\title{
Citric Acid-Assisted Preparation of Biochar Loaded with Cop- per/Nickel Bimetallic Nanoparticles
}

\author{
Jessim Omiri ${ }^{1}$, Youssef Snoussi ${ }^{1}$, Arvind K. Bhakta ${ }^{1,2}$, Stéphanie Truong ${ }^{1}$, Souad Ammar ${ }^{1}$, Ahmed M. Khalil ${ }^{1,3}$, Mo- \\ hamed Jouini and Mohamed M. Chehimi1,*
}

1. Université de Paris, CNRS, ITODYS (UMR 7086), Paris 75013, France

2. Department of Chemistry, St. Joseph's College, Bangalore, Karnataka, 560 027, India

3. Photochemistry Department, National Research Centre (NRC), Dokki, Giza 12622, Egypt

*Correspondence: mohamed.chehimi@cnrs.fr

\begin{abstract}
Biochar is a carbon allotrope obtained by pyrolysis of biomass, usually agro-waste. Owing to the demand for sustainable development, biochar is continuously raising much hope in the scientific community. However, in order to impart it with new properties, its modification is required, either in situ during pyrolysis, or after the carbonization process. Herein, we propose a new direct approach to obtain bimetallic copper/nickel nanoparticle-loaded on olive stone biochar. The bimetallic-coated biochar and the reference materials bare biochar, copper-loaded and nickel-loaded biochar were prepared at $400{ }^{\circ} \mathrm{C}$ under a stream of dinitrogen from olive pit powder particles impregnated first with citric acid (CA), and then with copper and nickel nitrates. We have employed citric acid in the process in order to check its effect on the structural and textural properties of biochar supporting the metallic nanoparticles. Surprisingly, citric acid induced the formation of agglomerated or even raspberry-shaped, bimetallic copper/nickel nanoparticles. Large 450-500 nm-sized agglomerates of $\sim 80 \mathrm{~nm}$ bimetallic CuNi NPs were noted for B-CA@CuNi. Interestingly, for biochar material prepared with initial $\mathrm{Cu} / \mathrm{Ni}=10$ molar ratio (B-CA@CuNi10/1), the bimetallic NPs formed unusual nano-raspberries $(174 \pm 8 \mathrm{~nm}$ in size) which are agglomerates of individual $10-20 \mathrm{~nm}$-sized $\mathrm{CuNi10/1}$ nanoparticles. The B-CA@CuNi and reference materials were characterized by Raman spectroscopy, scanning electron microscopy (SEM)/energy-dispersive X-ray spectroscopy (EDX), X-ray diffraction (XRD) and magnetometry. The B-CA@CuNi and B-CA@Ni materials could be attracted efficiently with a magnet, but not B-CA@CuNi10/1 due to a low nickel loading. B-CA@CuNi was tested as a catalyst for the degradation of methyl orange (MO). Discoloration was noted within 10 min, much faster than a similar material prepared in the absence of CA. B-CA@CuNi could be recycled at least 3 times with exhibit as fast discoloration catalysis performance.
\end{abstract}

This paper stresses the important role of citric acid in shaping the bimetallic nanoparticles loaded in situ on biochar during the slow pyrolysis process and to enable faster catalysed discoloration of organic dye solution.

Keywords: olea europaea; olive stone; agro-waste; slow pyrolysis; bimetallic nano-alloys; catalyzed dye degradation; Trash-to-Treasure

\section{Introduction}

One way to valorize agro-wastes is via pyrolysis into biochar [1], under inert atmosphere or in the presence of very low amount of air [2-4]. "Biochar" is nowadays a very hot research topic because it arises from the Trash-to-Treasure concept $[5,6]$, and thus falls within the general domain of sustainable development [7]. Much has been said on the preparation of biochar [8], and control of its composition [9], graphitization degree [10] and porosity [11] among other features [2, 12, 13]. Moreover, biochar could even be employed as a source of making novel graphene or carbon dots [14, 15], as well as carbon nanotubes [16]. However, in the recent years it has soon been realized that biochar could be engineered using additives during its preparation, or after carbonization process. For example, numerous reports grafting molecular [17] or macromolecular species [18] to biochar supports, or design metal oxide [19] and metallic nanoparticle-decorated biochar 
$[20,21]$. The applications [22] encompass, hydrogen evolution reaction and water splitting [23], removal of chromium VI [20], catalysed degradation of dyes [24], soil remediation [25], electrochemical energy storage [26], the design of support for N-fertilizers [27], reducing phosphorous loss [28], to name but a few.

Recently, we have proposed a simple way to make biochar loaded with copper/nickel bimetallic nanoparticles (B@CuNi) [21]. The rationale for fabricating CuNi bimetallic NPs on biochar lies in the interest of this low-cost, magnetic, bimetallic nanomaterial in the catalysis of nitrate reduction [29, 30], reduction of $\mathrm{Cr}$ (VI) to $\mathrm{Cr}$ (III) [31], oxygen evolution and reduction reactions [32] or degradation of nitrophenol and organic dyes [33]. When directly coated on biochar during pyrolysis, CuNi bimetallic NPs were found to be 10-20 nm-sized and uniformly dispersed over the biochar surface [21]. The composite material was found to be magnetic and could catalyse the degradation of methyl orange (MO) within 30 minutes [21].

In order to increase the porosity of the underlying biochar, one can pyrolyze the initial agrowaste modified with carbonates or oxalates, but also with carboxylic acids such as citric acid. For example, incorporation of carboxylic groups using citric acid monohydrate followed by fixation of $\mathrm{K}^{+}$ions via ion exchange with $\mathrm{KOH}$ and then carbonization at elevated temperature resulted in biochar with micropores [34, 35]. A study was carried out to understand the effect of various potassium containing compounds for porous carbons. Mixing of rice husk biomass with these compounds via ball milling followed by heating causes catalytic pyrolysis with the decreasing order $\mathrm{KOH}>\mathrm{K}_{2} \mathrm{CO}_{3}>\mathrm{K}_{2} \mathrm{C}_{2} \mathrm{O}_{4}$ [36]. Bridging the gap with our ongoing research work, one could expect the tricarboxylic group from citric acid to play crucial role in the bi- and tri-valent metal ions complex formation [37].

In our protocol [21], wet impregnation of the agro-waste powder prior to pyrolysis ensured remarkable distribution of the dispersed bimetallic nanoparticles. Therefore, we reasoned that it is beneficial to impregnate the biomass first with citric acid prior to addition of copper and/or nickel nitrates and thus investigate the effect of citric acid on the properties of the biochar and the supported nanoparticles. Surprisingly, scanning electron microscopy (SEM) imaging revealed the deposition of agglomerated nanoparticles and even some were found to be raspberry-shaped, which is quite unusual, and thus worth to be reported. Indeed, and for the very best of our knowledge, no previous study considered combining carboxylic acid treatment of biomass for the deposition of bimetallic nanoparticles, hence the motivation for this work.

Herein, we report on the preparation of olive pit biochar loaded with copper/nickel bimetallic nanoparticles by slow pyrolysis, at $400{ }^{\circ} \mathrm{C}$. The initial agro-waste powder was first impregnated with citric acid (CA) then loaded with copper and nickel nitrates prior to pyrolysis. The final citric acid-impregnated OP biochar (B) loaded with bimetallic copper-nickel (B-CA@CuNi) and the reference materials B-CA, B-CA@CuNi10/1 and B-CA@Ni were characterized by scanning electron microscopy (SEM)/ energy dispersive X-ray spectroscopy (EDX), X-ray diffraction (XRD), Raman and magnetization measurements employing superconducting quantum interference device (SQUID). The final B-CA@CuNi was tested as potential catalyst for the degradation of Methyl Orange (MO) dye, taken as model organic pollutant.

\section{Experimental}

\subsection{Chemicals}

Olive pit (OP) powder (grain diameter from 40 to $600 \mu \mathrm{m}$ [38]) was obtained after extraction of Tunisian olive oil (see details in [21]). Citric acid (CA), metal nitrate salts, $\mathrm{Cu}\left(\mathrm{NO}_{3}\right)_{2}, 3 \mathrm{H}_{2} \mathrm{O}$ and $\mathrm{Ni}\left(\mathrm{NO}_{3}\right)_{2}, 6 \mathrm{H}_{2} \mathrm{O}$, sodium hydroborate $\left(\mathrm{NaBH}_{4}\right)$, and Methyl Orange (MO) were purchased from Aldrich and used as received. We have used distilled water to dissolve citric acid and the metal nitrates prior to pyrolysis, and to prepare aqueous solutions for dye degradation tests. 


\subsection{Preparation of biochar materials}

OP particles were impregnated with an aqueous CA solution in the nominal ratio 1 mmol CA/1g OP using a minimal amount of water (see Table 1). The powder was dried at $70{ }^{\circ} \mathrm{C}$ and mixed thoroughly with a spatula. The same CA-loaded OP powder was impregnated again with a mixture of $\mathrm{Cu}\left(\mathrm{NO}_{3}\right)_{2}, 3 \mathrm{H}_{2} \mathrm{O}$ and/or $\mathrm{Ni}\left(\mathrm{NO}_{3}\right)_{2}, 6 \mathrm{H}_{2} \mathrm{O}(1 \mathrm{mmol}$ each per $1 \mathrm{~g}$ of $\mathrm{OP})$. Additionally, we have prepared a biochar loaded with metallic nanoparticles from $10 / 1$ initial molar ratio of $\mathrm{Cu}\left(\mathrm{NO}_{3}\right)_{2}, 3 \mathrm{H}_{2} \mathrm{O} / \mathrm{Ni}\left(\mathrm{NO}_{3}\right)_{2}, 6 \mathrm{H}_{2} \mathrm{O}$. The mixture containing CA-impregnated OP, copper and nickel nitrates was left to dry overnight, then thoroughly mixed, dried again and ground in agate mortar. It was weighed several times and mixed until the weight was stable.

Visually, the OP powder changes color upon impregnation with CA and further with the metal nitrates (this is detailed below in section 3). About 0.8 to $1 \mathrm{~g}$ impregnated olive pit particles were pyrolyzed under $\mathrm{N}_{2}$ stream for $2 \mathrm{~h}$ at $400{ }^{\circ} \mathrm{C}$, in a tubular furnace. The apparatus was stopped for cooling, however under $\mathrm{N}_{2}$ flow in order to avoid oxidation and ash formation.

The experimental conditions for preparing the impregnated biomass and pyrolysis are reported in Table 1.

\subsection{Characterization}

X-ray diffraction (XRD) patterns of the biochar samples were acquired with an $X^{\prime}$ Pert-Pro Panalytical diffractometer, fitted with a Cobalt X-ray source $(\lambda=1.7889 \AA)$ and operating in the Bragg-Brentano reflexion geometry (tube current $=40 \mathrm{~mA}$ and operating voltage $=40 \mathrm{kV}$ ).

Raman spectra of the biochar specimens were recorded using Horiba HR 800 instrument, operating with a wavelength of the He-Ne laser beam set of $633 \mathrm{~nm}$.

SEM observations and EDX spectral acquisition were performed on a Gemini SEM 360.

The magnetic properties of the produced catalysts were measured by a Quantum Design physical properties measurement system (PPMS). Typically, the dc-magnetization $M$ was measured by cycling the magnetic field $\mu_{0} \mathrm{H}$ between +7 and $-7 \mathrm{~T}$ at room temperature, $300 \mathrm{~K}$. The diamagnetic contribution of the biochar was then subtracted from the recorded $M\left(\mu_{0} H\right)$ data before to be plotted for the Ni-based catalysts, expressed per gram of catalyst.

\subsection{Catalytic degradation of Methyl Orange.}

$1 \mathrm{mg}$ of catalyst was added to a beaker containing $4 \mathrm{~mL}$ of $20 \mathrm{ppm}$ aqueous dye solution. The beaker was sonicated for $2 \mathrm{~min}$; then $30 \mathrm{mg}$ of $\mathrm{NaBH}_{4}$ were poured in the beaker in order to trigger the catalysed reaction. A strong magnet was placed near the beaker to attract the biochar and be able to sample sufficient MO solution for UV-vis analysis. Typically, the MO concentration decrease was monitored using a Perkin ElmerLambda 1050 spectrophotometer, operated in the transmission mode (optical length $1 \mathrm{~cm}$ ). The optical absorption spectra of the reaction solution containing $\mathrm{MO}$, were recorded in the $200-800 \mathrm{~nm}$ range.

Table 1. Experimental conditions for the preparation of impregnated biomass and corresponding biochar.

\begin{tabular}{|c|c|c|c|c|c|c|c|c|}
\hline Materials & $\begin{array}{l}\text { OP mass } \\
\pm 0.0001 \\
(\mathrm{~g})\end{array}$ & $\begin{array}{c}\text { OP mass } \\
\text { after } \\
\text { impregnation } \\
\pm 0.0001(\mathrm{~g}) \\
\end{array}$ & $\begin{array}{c}\text { CA mass } \\
\pm 0.0001(\mathrm{~g})\end{array}$ & $\begin{array}{l}\mathrm{Cu}\left(\mathrm{NO}_{3}\right)_{2} \cdot 3 \\
\mathrm{H}_{2} \mathrm{O} \text { mass } \\
(\mathrm{g}) / \mathrm{mmol}\end{array}$ & $\begin{array}{c}\mathrm{Ni}\left(\mathrm{NO}_{3}\right)_{2} \cdot 6 \mathrm{H}_{2} \mathrm{O} \\
\text { mass } \\
(\mathrm{g}) / \mathrm{mmol}\end{array}$ & $\begin{array}{c}\text { Pyrolyzed OP } \\
\text { impregnated } \\
\text { with metal ions } \\
\text { (g) }\end{array}$ & $\begin{array}{c}\text { Biochar mass } \\
\text { (g) and yield } \\
(\%)\end{array}$ & $\begin{array}{c}\text { Final metal } \\
\text { /biochar } \\
\text { Ratio } \\
\text { (mmol/g) } \\
\end{array}$ \\
\hline $\mathrm{OP}+\mathrm{CA}$ & 3.0000 & 3.5762 & 0.5762 & - & - & 0.7750 & $0.220(28.4 \%)$ & - \\
\hline $\begin{array}{c}\mathrm{OP}+\mathrm{CA}+ \\
\mathrm{CuNi}\end{array}$ & 3.0010 & 5.1750 & 0.5760 & $0.7250 / 3.000$ & $0.8730 / 3.002$ & 0.8010 & $0.246(30.7 \%)$ & $3.776 \mathrm{mmol} / \mathrm{g}$ \\
\hline
\end{tabular}




\begin{tabular}{|c|c|c|c|c|c|c|c|c|}
\hline $\begin{array}{c}\mathrm{OP}+\mathrm{CA}+ \\
\mathrm{CuNi}(10 / 1)\end{array}$ & 3.0060 & 4,4045 & 0.5775 & $0.7245 / 2.999$ & $0.0965 / 0.332$ & 0.7960 & $0.254(31.9 \%)$ & $2.37 \mathrm{mmol} / \mathrm{g}$ \\
\hline $\begin{array}{c}\mathrm{OP}+\mathrm{CA}+\mathrm{Ni} \\
\neq\end{array}$ & 3.0093 & 4.4586 & 0.5760 & - & $0.8733 / 3.003$ & 0.8010 & $0.269(33.6 \%)$ & $2.30 \mathrm{mmol} / \mathrm{g}$ \\
\hline
\end{tabular}

Water volume for impregnation of citric acid $=20 \mathrm{~mL}$, and for impregnation of nitrates $=20 \mathrm{~mL}$. Pyrolysis conditions $=30^{\circ} \mathrm{C} / \mathrm{min}$; $\mathrm{T}_{\max }=400^{\circ} \mathrm{C}$; Resident at $\mathrm{T}_{\max }=2 \mathrm{~h}$.

\section{Results and Discussion}

Figure 1 displays the main steps in the preparation of biochar materials from the biomass (Figure 1a), and the digital photographs of the materials (Figure 1b). Wet impregnation by citric acid and by the nitrates ensures uniform impregnation prior to pyrolysis as could be noted from the digital picture. All metallic nanoparticle-coated biochar look the same, but for the sake of simplicity only the B-CA@CuNi is shown.



Figure 1. (a) General pathway for the design of biochar materials; and (b) digital photographs of OP grains before and after impregnation with citric acid, and copper/nickel nitrates (in three proportions) leading to black biochar composite powder.

\subsection{Physicochemical properties of biochar nanocomposites}

\subsubsection{Structural analysis studies by XRD and Raman spectroscopy}

Figure 2 displays XRD patterns of B-CA based catalysts, B-CA and B@CuNi prepared without any CA. The diffraction peak positions were compared to those tabulated for $\mathrm{Cu}$ and $\mathrm{Ni}$, and their oxides. (111) and (200) plans of the metallic phases are indicated by dashed $(\mathrm{Cu})$ or solid $(\mathrm{Ni})$ black vertical lines.

The amorphous nature of the underlying carbon substrate is evidenced by the featureless pattern of the metal-free B-CA sample. In contrast, the $\mathrm{Cu}$ and/or Ni-loaded samples exhibit broadened peaks at $2 \theta$ positions matching, for some of them, those tabulated for bulk Cu (ICDD n98-005-2256) and/or Ni (ICDD n $\left.{ }^{\circ} 98-004-1508\right)$. This means that the produced metallic particles are very small in size and that they are partly oxidized. Additionally, the presence of the organic acid during the material processing seems to lead to the formation of metallic crystals that are smaller in size than those prepared from the same metal nitrates but without any CA [21]. Particularly, the nickel richest particles are much smaller, and thus much more sensitive to oxidation upon air exposure (see Table 2). 
Table 2. Metal and metal oxide weight ratio and crystal size inferred from diffraction peak intensity and broadening. ${ }^{\text {. }}$

\begin{tabular}{|c|c|c|c|c|}
\hline Catalyst & Crystal phase & Phase content (wt.-\%) & Crystal size (̊̊) & Ref. \\
\hline \multirow{2}{*}{ B-CA@Ni } & $\mathrm{Ni}$ & 30 & 30 & This work \\
\hline & $\mathrm{NiO}$ & 70 & 60 & \\
\hline \multirow{4}{*}{ B-CA@CuNi } & $\mathrm{Cu}$ & 48 & 130 & \\
\hline & $\mathrm{Cu}_{2} \mathrm{O}$ & 12 & 125 & \\
\hline & $\mathrm{Ni}$ & 10 & 40 & \\
\hline & $\mathrm{NiO}$ & 30 & 75 & \\
\hline \multirow{4}{*}{ B-CA@CuNi10/1 } & $\mathrm{Cu}$ & 67 & 120 & \\
\hline & $\mathrm{Cu}_{2} \mathrm{O}$ & 21 & 110 & \\
\hline & $\mathrm{Ni}$ & 10 & 40 & \\
\hline & $\mathrm{NiO}$ & 2 & 60 & \\
\hline \multirow[t]{2}{*}{$\mathrm{B} @ \mathrm{CuNi}$} & $\mathrm{Cu}$ & 70 & 190 & [21] \\
\hline & $\mathrm{Ni}$ & 30 & 40 & \\
\hline
\end{tabular}

a Hypothesis: isotropic shapes are considered for all catalysts prepared in the presence of CA, compared to those of $\mathrm{B} @ \mathrm{CuNi} 1 / 1$ catalyst produced without any pre-impregnation of OP with CA.

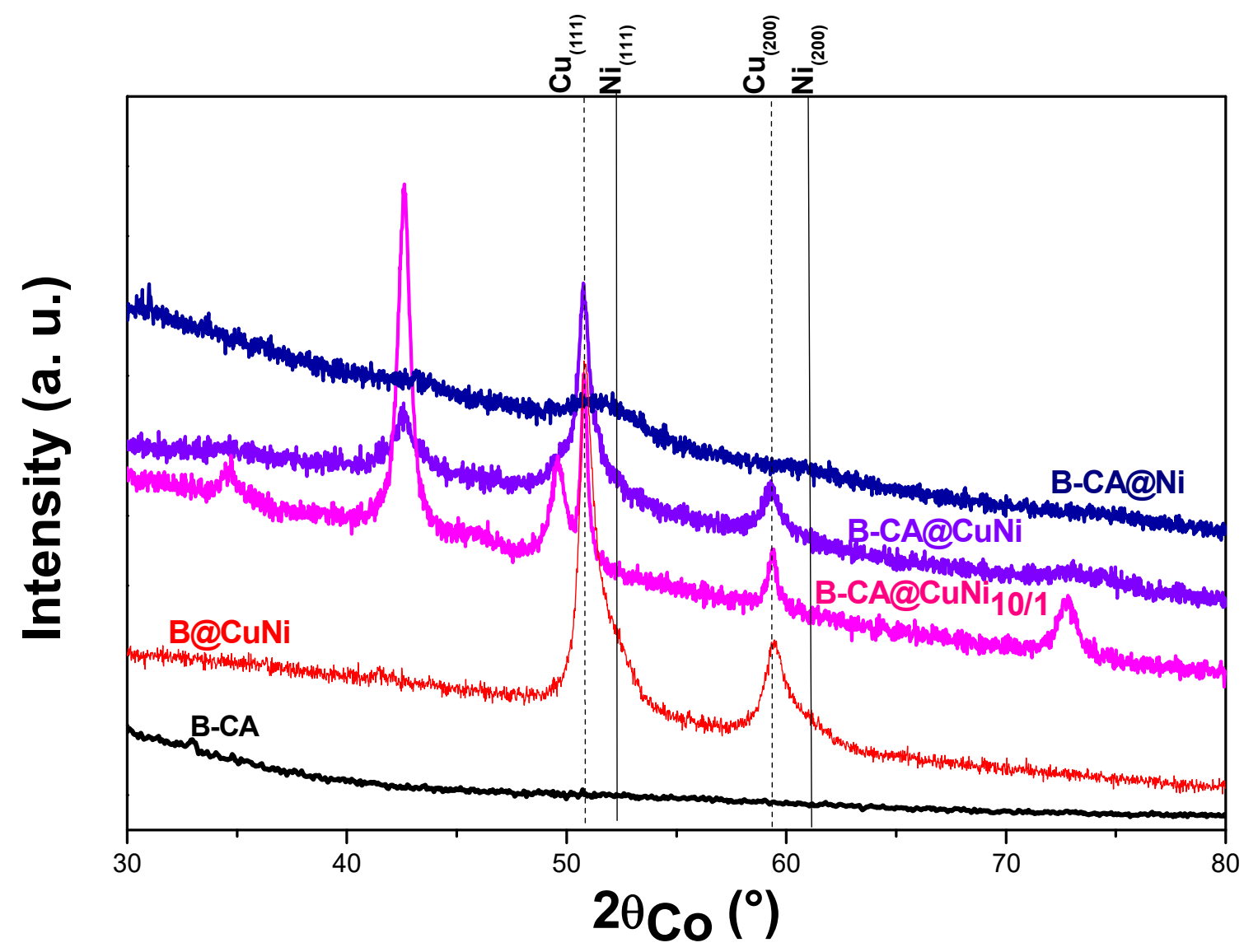

Figure 2. XRD patterns of B-CA@CuNi, B-CA@CuNi10/1 and B-CA@Ni compared to those of metal free B-CA and CA-free $\mathrm{B} @ \mathrm{CuNi} / 1 / 1$ samples.

Peak broadening did not allow evidencing a net shift of the peak position to confirm

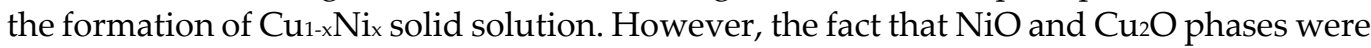
identified in the recorded patterns for all the produced B-CA based catalysts, disagrees with this hypothesis. Clearly, the use of CA induces cation complexation over the whole OP surface, possibly nickel cations much more than copper ones, which leads to increased metal nucleation sites during pyrolysis, therefore generating very small metal crystals. The differences in affinities between each type of cation and citric acid contribute to the 
segregation of their related metallic phases. This feature is not at all relevant when catalysts synthesis proceeds without CA. Indeed, B@CuNi XRD pattern matches much more with the signature of larger in size $\mathrm{Cu}$ - and $\mathrm{Ni}$-rich $\mathrm{Cu}_{1-x \mathrm{Ni}}$ solid solution crystals, with peak positions slightly comparable to those tabulated for pure $\mathrm{Cu}$ and pure $\mathrm{Ni}$, respectively [21].

To summarize, most probably citric acid is prone to provide ultrafine metallic NPs, which get oxidized upon air exposure. This oxidation is not so critical, since the metallic phase can be easily recovered by adding sodium borohydride $\mathrm{NaBH}_{4}$ during the catalysis assays.

To complete these copper-nickel structural investigations, the Raman spectra of all the produced composites were recorded to characterize the biochar support (Figure 3).

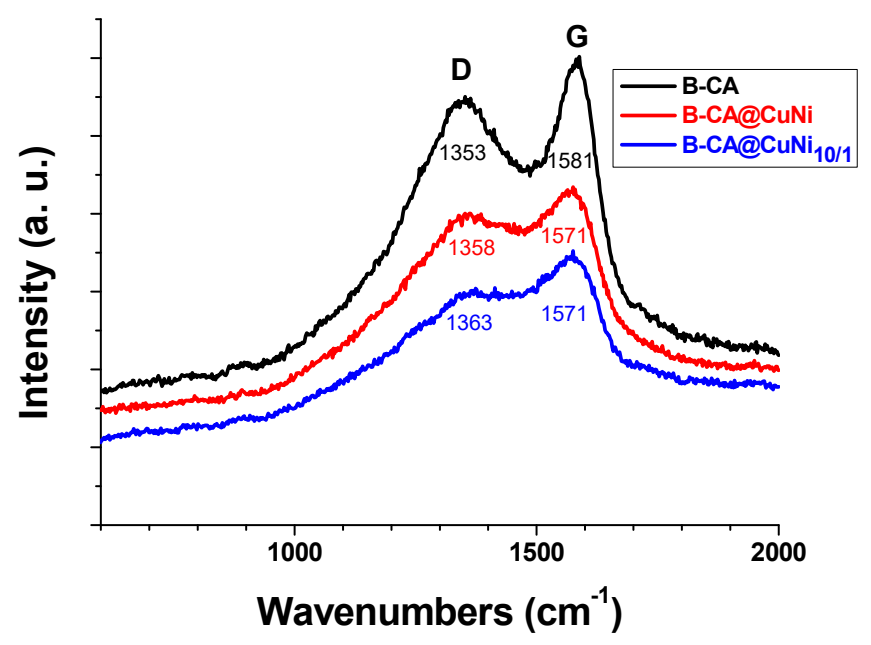

Figure 3. Raman spectra of B-CA, B-CA@CuNi, and B-CA@CuNi10/1.

Interestingly, all spectra exhibit doublets characteristic of carbon-based matter. Indeed, two peaks coined D and G, are centred at $1351 \pm 1$ and $1576 \pm 5 \mathrm{~cm}^{-1}$, respectively (Figure 3) [39]. The D band accounts for defects in the biochar chemical structure, for example $\mathrm{C}-\mathrm{C}$ bonds between aromatic rings [40] as well as $\mathrm{C}-\mathrm{O}$ defects [39]. The $\mathrm{G}$ band is assigned to aromatic ring breathing and $\mathrm{C}=\mathrm{C}$ bonds. The $\mathrm{D} / \mathrm{G}$ intensity ratio (peak height ratio) are in the $0.79-0.89$ range, higher than 0.67 obtained for the same biochar prepared under slightly different conditions, at $400^{\circ} \mathrm{C}$ under $\mathrm{N}_{2} / \mathrm{H}_{2}$ mixture [21], and also higher than 0.58 obtained for Spanish olive stones carbonized at $600^{\circ} \mathrm{C}$ [39]. The slightly higher D/G ratio indicates increase in defects. Herein, D/G ratio remains quasi the same but one can note that the doublets are less resolved in the case of supported nanocatalysts. This suggests possible interactions between the biochar support and the immobilized nanocatalysts, or possible metal-induced defects during pyrolysis. Indeed, the spectral region between D and $\mathrm{G}$ bands, coincides with $\mathrm{V}$ bands assigned to $\mathrm{sp}^{2}$ and sp3 amorphous carbonaceous structures [41]. Convolution of the V, D and G bands yields less resolution and decrease in the peak position difference (G-D) from $228 \mathrm{~cm}^{-1}$ for B-CA to 213 (B-CA@CuNi) and 208 cm$^{-1}$ (B-CA@CuNi10/1).

\subsubsection{Surface morphology and elemental composition (SEM/EDX)}

Figure 4 shows SEM pictures of B-CA, B-CA@Ni, B-CA@CuNi, and B-CA@CuNi10/1. Besides a few pores at the surface B-CA ( 400 nm diameter), the CA-treated biomass yields a biochar with wax-like surface (Figure 4a), similar to the OP biochar prepared at $400{ }^{\circ} \mathrm{C}$ for $15 \mathrm{~min}$ (see for example [21]). For B-CA@Ni (Figure 4b), about $100 \mathrm{~nm}$-sized, spherical Ni NPs are visible. The NPs are dispersed all over the biochar surface but show substantial agglomeration. Figures 4c-e display SEM images of B-CA@CuNi at various 
magnifications; the biochar shows particles with undefined shapes (Figure 4c), with good dispersion of CuNi NPs (Figure 4d). Higher magnification shows localized $400 \mathrm{~nm}$-sized spherical agglomerates (Figure 4e). Similar good dispersion of bimetallic NPs is noted for B-CA@CuNi10/1 (Figure 4f). Zooming in permits to note raspberry-shaped CuNi10/1 NPs having $174 \pm 8 \mathrm{~nm}$ size (Figure $4 \mathrm{~g}$ ).

EDX spectra are displayed in Figure 5. Only C, O and $\mathrm{Ni}$ peaks are noted for B$\mathrm{CA} @ \mathrm{Ni}$ (Figure 5a), but both $\mathrm{Cu}$ and $\mathrm{Ni}$ are visible at similar relative peak intensity for BCA@CuNi (Figure 5b). In the case of 10-fold higher initial copper nitrate impregnation compared to nickel nitrate, the final B-CA@CuNi10/1 exhibits very small Ni peak compared to that of $\mathrm{Cu}$ (Figure 5c).

The $\mathrm{Cu} / \mathrm{Ni}$ atomic ratio is found to be $1.18 \pm 0.08$ and $6.61 \pm 0.72$ for B-CA@CuNi and B-CA@CuNi10/1 composites, respectively. Whilst for the equal molar impregnation, $\mathrm{Cu} / \mathrm{Ni}$ is close to 1 for B-CA@CuNi, the B-CA@CuNi10/1 composite shows about 30\% lower final $\mathrm{Cu} / \mathrm{Ni}$ atomic ratio, possibly due to the existence of nickel-richest NPs, as judged from XRD studies (Section 3.1.1). 


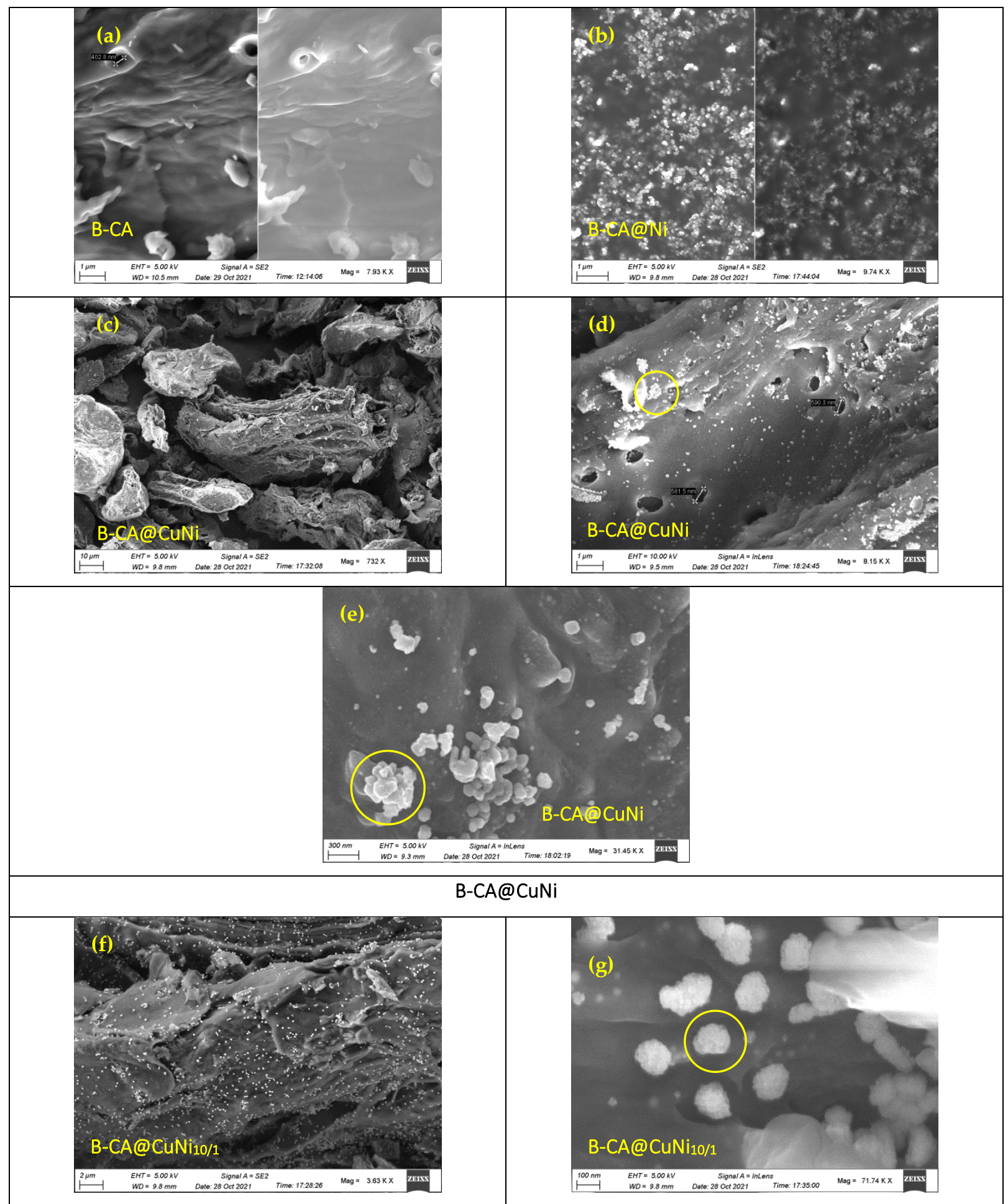

Figure 4. SEM pictures of B-CA (a), B-CA@Ni (b), B-CA@CuNi (c-e), and B-CA@CuNi10/1 (f,g). 



$\mathrm{X}$-ray energy (keV)

Figure 5. EDX spectra of B-CA@Ni (a), B-CA@CuNi (b), and B-CA@CuNi10/1 (c).

\subsubsection{Magnetic properties of biochar materials}

The variation of the magnetization as a function of the magnetic field at RT is plotted for the three nickel-based biochar catalysts B-CA@CuNi, B-CA@CuNi10/1 and B-CA@Ni (Figure 6). A typical soft ferromagnetic behavior can be noted with a high field magnetization which decreases when the Ni content decreases, explaining why the nickel-richest biochar are more easily attracted by an external magnetic field during the catalysis assays.

Interestingly, expressed per gram of nickel, the obtained high-field magnetization values are significantly lower than the expected $50 \mathrm{emu} / \mathrm{g}$ of bulk $\mathrm{Ni}$, even for the copperfree, Ni-based catalyst (B-CA@Ni). This means that the major part of the involved nickel species corresponds to $\mathrm{Ni}^{2+}$ instead of $\mathrm{Ni}^{0}$, without degrading their catalytic performances. This result agrees with those inferred from XRD and SEM/EDX analyses, which clearly show that nickel is oxidized on the biochar surface, most probably upon exposure to air, following the pyrolysis process. It seems that the operating catalysis conditions, which require the introduction of $\mathrm{NaBH}_{4}$ reducing agent into the reaction medium, allow reducing in situ the $\mathrm{Ni}^{2+}$ cations into $\mathrm{Ni}^{0}$, making the amount of available metal nickel surfaces high enough to conduct the desired catalytic reaction.

Nonetheless, as noted in the video (Supplementary Material), B-CA@CuNi is attracted by the magnet to the sidewall of the beaker, and returns to the center of the beaker upon withdrawal of the magnet. In contrast, B-CA@CuNi10/1 is not attracted due to the low nickel loading on the biochar. 




Figure 6. Magnetization of metallic nanoparticle-coated biochar versus the magnetic field, at RT.

\subsection{Potential application: degradation of methyl orange}

The catalytic performance of B-AC@CuNi was tested in the degradation of methyl orange (MO), chosen as a model reaction (Figure 7). As a first "Yes-No" test, Figure 7a displays the MO UV-vis spectrum before and after catalytic discoloration resulting from the degradation of the dye. Discoloration was achieved indeed in less than $10 \mathrm{~min}$, much faster than the 30 minutes reported for the same type of composite but prepared in the absence of citric acid [21]. Yet, this Yes-No test was done with excess $\mathrm{NaBH}_{4}$.

In order to establish the kinetic study and be able to monitor the variation of the organic molecule concentration versus time, measurements were performed with the following set: $1 \mathrm{mg}$ of the catalyst was dispersed in $10 \mathrm{ml}$ of $\mathrm{MO}$ solution $\left(20 \mathrm{mg} \cdot \mathrm{L}^{-1}\right.$, or 20 $\mathrm{ppm}$ ) before adding $50 \mu \mathrm{L}$ of sodium borohydride solution (2M) which corresponds to the time to. The final mass of $\mathrm{NaBH}_{4}$ introduced to the reaction medium was $3.8 \mathrm{mg}$. However, $3.8 \mathrm{mg}$ are herein introduced in $10 \mathrm{~mL}$ instead of $30 \mathrm{mg}$ in $4 \mathrm{~mL}$ for the fast Yes-No test (about 8-fold less mass, or 20-fold lower concentration of sodium borohydride).

As one can see in Figure $7 \mathrm{~b}$, as the orange color is vanishing, so the intensity of the absorption peak at $463 \mathrm{~nm}$ is decreasing. In contrast, one can note the intensity increase of the peaks centred at $247 \mathrm{~nm}$ and $\sim 280 \mathrm{~nm}$. These two last peaks could be assigned to the sulfanilic acid and the $\mathrm{N}, \mathrm{N}$-dimethylaniline, respectively, which are the two catalyzed degradation products of the dye.

It was practically difficult to collect $1 \mathrm{mg}$ of the catalyst even though its high magnetic properties. To proceed with the reusability of $1 \mathrm{mg}$ of B-AC@CuNi, each time $10 \mathrm{ml}$ of the MO solution and $50 \mu \mathrm{L}$ of $\mathrm{NaBH}_{4} 2 \mathrm{M}$ solution were added after the total disappearing of the orange initial color until reaching 3 successive cycles. The final solution color turned transparent accompanied by a total degradation of the azo dye (See dashed black line in Figure $7 b)$.

The evolution of the ratio $\mathrm{C} / \mathrm{C}_{0}$ versus $\mathrm{t}$ shown in Figure $7 \mathrm{c}$ (and deduced from the processed peaks in Figure $7 \mathrm{~b}$ ) is characterized by three main phases: (i) fast degradation rate in the first $20 \mathrm{~min}$ where almost $45 \%$ of MO have been degraded, (ii) then the rate became slower until $60 \mathrm{~min}$ and the degradation efficiency reached $70 \%$, and (iii) finally turning to very slow during in the last $90 \mathrm{~min}$ (75\% is the final degradation yield). 
The fitting of the experimental data to the pseudo-first order and the pseudo-second order rate laws showed that the data do not fit the expected pseudo-first order, but excellently fit the pseudo-second order model. It may be recalled that the linearized equations of the two models are given by:

$$
\begin{aligned}
& \ln \frac{C}{C_{0}}=-K_{1} t(1) \\
& \text { and, } \\
& \frac{t}{q_{t}}=\frac{1}{K_{2}}+\frac{t}{q_{e}} \text { (2) }
\end{aligned}
$$

For the pseudo-first order(equation (1)), $K_{1}$ is the apparent rate constant, $C_{0}$ is the initial concentration of $\mathrm{MO}$ and $C$ is the concentration of the dye at time $t$. For the pseudosecond order (equation (2)), $K_{2}$ is the equilibrium constant rate (the adsorption of MO over B-AC@CuNi), $q_{t}$ and $q_{e}$ are the adsorbed amount at time $t$ and at the equilibrium time, respectively.

The analyses of the experimental data according the rate laws are displayed in Figures $7 \mathrm{~d}, \mathrm{e}$.
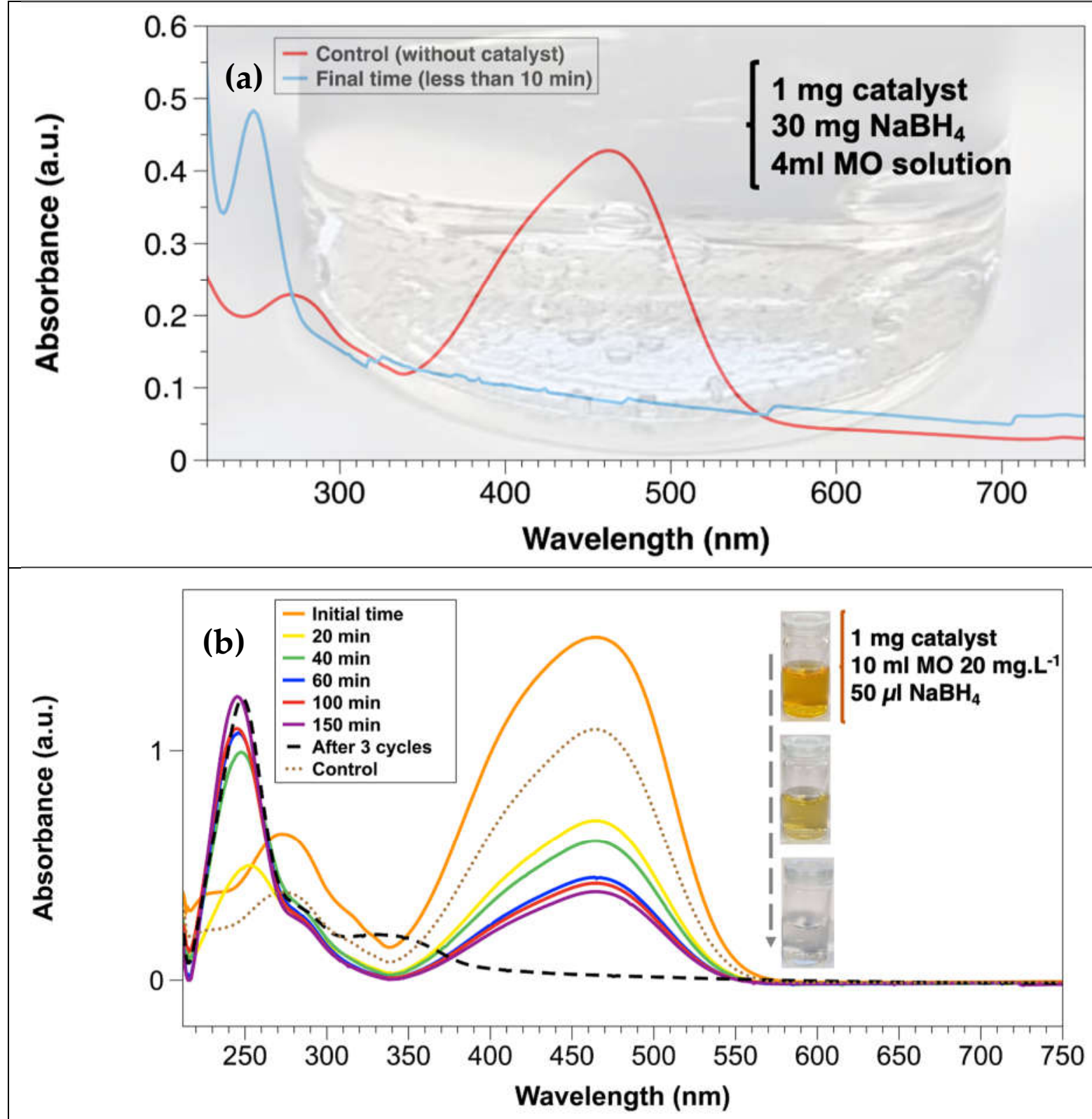


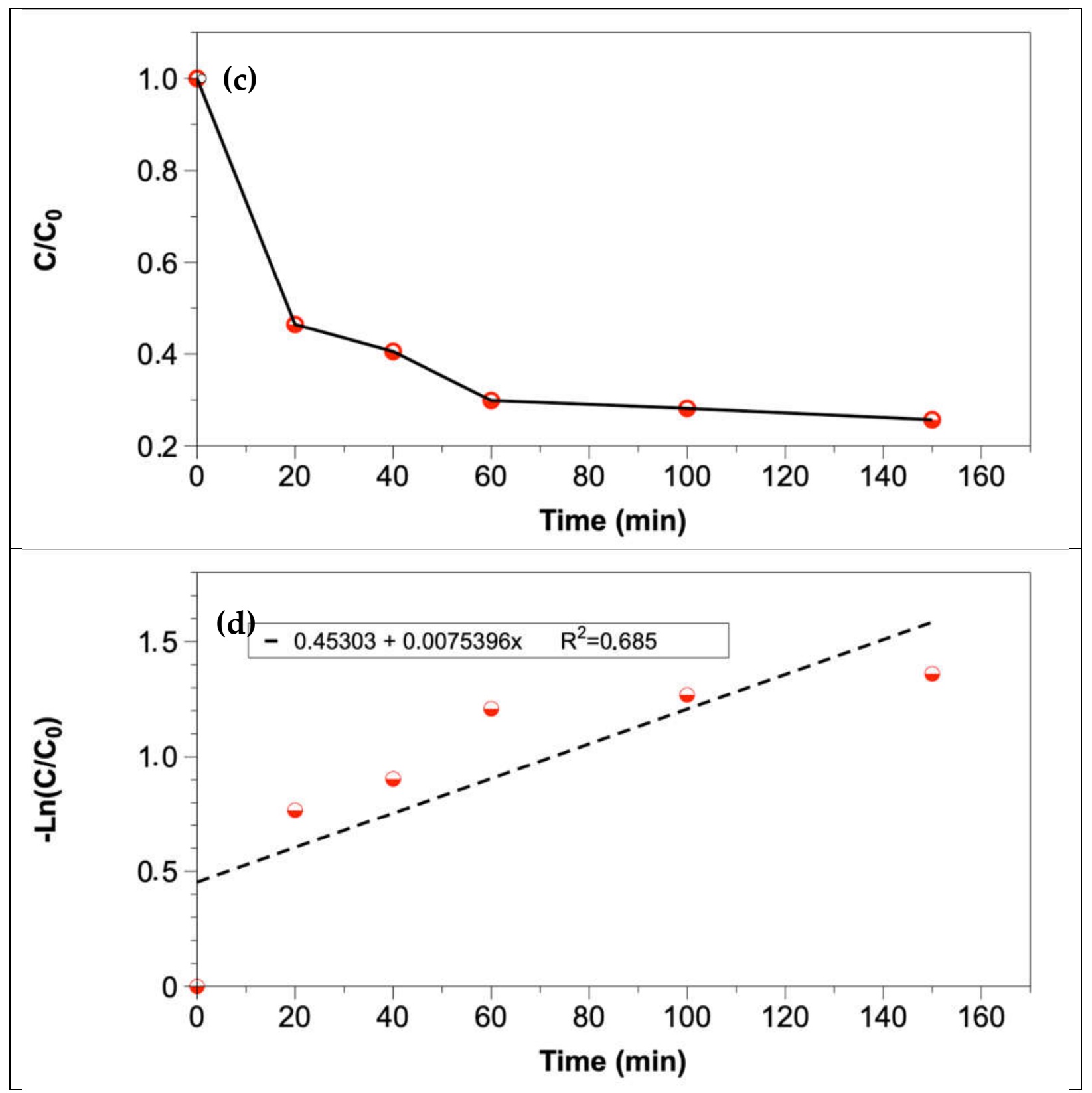






Figure 7. Kinetic study of MO degradation: (a) fast test using excess $\mathrm{NaBH}_{4}\left(7.5 \mathrm{mg} \cdot \mathrm{mL}^{-1}\right)$, (b) stacked UV-vis spectra recorded using low $\mathrm{NaBH}_{4}$ concentration (0.38 mg.mL-1), (c) $\mathrm{C} / \mathrm{CO}$-vs-time plot for MO degradation, (d) pseudo-first order kinetics, and (e) pseudo-second order kinetics of catalysed MO degradation.

A proposed degradation mechanism of $\mathrm{MO}$ in presence of $\mathrm{NaBH}_{4}$ is illustrated in Figure 8. It is a two-step mechanism where the antibonding orbital of $\mathrm{H}_{2}$, generated after the reaction of sodium borohydride and water, reacts with the unfilled d-bond of the metal to form a dative bonding (between $\mathrm{H}_{2}$ and the metallic nanostructure).



Figure 8. Proposed mechanism of degradation of $\mathrm{MO}$ using B-CA@CuNi in the presence of $\mathrm{NaBH}_{4}$. 


\section{Conclusion}

Olive pit (OP) grains loaded with $\mathrm{CuNi}$ bimetallic nanoparticles were prepared by slow pyrolysis at $400^{\circ} \mathrm{C}$, under dinitrogen stream for $2 \mathrm{~h}$. In this follow-up to our previous work [21], we have investigated the effect of pre-impregnation of OP grains with citric acid (CA) on the structural and morphological properties of the final biochar, noted BCA@CuNi. Contrary to the previous findings, herein CA induces the formation of agglomerated metallic nanoparticles of either alloy or nickel NPs. Particularly, unusual raspberry-shaped nickel NPs were obtained in the case of B-CA@Ni. Moreover, more defects are noted for B-CA compared to the biochar prepared without CA as judged from the higher corresponding D/G ratio determined by Raman spectroscopy. On the crystallographic viewpoint, small bimetallic NPs are formed, some with nickel-rich phase. Small size readily induces metal oxidation of the supported NPs upon air exposure. However, not only B-CA@CuNi remains to be attracted by a magnet, but is also able to catalyse the degradation of Methyl Orange in the presence of $\mathrm{NaBH}_{4}$. This compound could help to reduce the formed metal oxides whilst it participates to the catalysed reaction. Discoloration of the dye was achieved with $1 \mathrm{mg}$ of B-CA@CuNi and in the presence of a low amount of $\mathrm{NaBH}_{4}$. It was monitored by UV-vis spectrometry and was not found, unexpectedly, to fit a pseudo-first order kinetics model.

This work conclusively shows that citric acid has not only altered the properties of the biochar, but induced unusual structures of metallic or bimetallic copper/nickel or pure nickel nanocatalysts.

Supplementary Materials: : https://vimeo.com/563459182

Author Contributions: Conceptualization (MMC, AMK, SA); Methodology (MMC, AMK, SA); Validation (all authors); Formal Analysis and Investigation (all authors); Writing-Original Draft (JO and MMC); Writing - Review \& Editing (all authors); Resources (MMC, SA); Supervision (MMC); Funding acquisition (AMK, AKB, SA, MMC).

Funding: A.M.K. and M.M.C. would like to thank both the French and Egyptian Governments for funding AMK's contribution through a fellowship granted by the French Embassy in Egypt (Institut Francais d'Egypte) and Science and Technology Development Fund (STDF)-Egypt, Project number (42248). Also, AKB is thankful to bourse WBI Excellence World ( $\mathrm{N}^{\circ}$ Imputation- 101386, and Article Budgétaire-33.01.00.07).

Acknowledgements: The authors would like to thank Mrs S. Gam-Derouich (Experimental officer) for SEM/EDX analyses.

Institutional Review Board Statement: Not applicable.

Informed Consent Statement: Not applicable.

Data Availability Statement: Original data available on request. They are not archived in any repository.

Conflicts of Interest: The authors declare no conflict of interest.

\section{References}

1. Nanda, S., et al., Biochar as an exceptional bioresource for energy, agronomy, carbon sequestration, activated carbon and specialty materials. Waste and Biomass Valorization, 2016. 7(2): p. 201-235.

2. Tripathi, M., J.N. Sahu, and P. Ganesan, Effect of process parameters on production of biochar from biomass waste through pyrolysis: A review. Renewable and Sustainable Energy Reviews, 2016. 55: p. 467-481.

3. Harussani, M. and S. Sapuan, Development of Kenaf Biochar in Engineering and Agricultural Applications. Chemistry Africa, 2021: p. 1-17.

4. Atabani, A., et al., A state-of-the-art review on spent coffee ground (SCG) pyrolysis for future biorefinery. Chemosphere, 2022. 286: p. 131730 . 
5. Sutar, S., P. Patil, and J. Jadhav, Recent advances in biochar technology for textile dyes wastewater remediation: A review. Environmental Research, 2022: p. 112841.

6. Lu, Y., et al., Turning Trash into Treasure: Pencil Waste-Derived Materials for Solar-Powered Water Evaporation. Energy Technology, 2020. 8(10): p. 2000567.

7. Kumar, A. and T. Bhattacharya, Biochar: a sustainable solution. Environment, Development and Sustainability, 2021. 23(5): p. 6642-6680.

8. Pan, X., et al., Preparation of biochar and biochar composites and their application in a Fenton-like process for wastewater decontamination: A review. Science of The Total Environment, 2021. 754: p. 142104.

9. Al-Wabel, M.I., et al., Pyrolysis temperature induced changes in characteristics and chemical composition of biochar produced from conocarpus wastes. Bioresource technology, 2013. 131: p. 374-379.

10. Eshun, J., et al., Characterization of the physicochemical and structural evolution of biomass particles during combined pyrolysis and CO2 gasification. Journal of the Energy Institute, 2019. 92(1): p. 82-93.

11. Leng, L., et al., An overview on engineering the surface area and porosity of biochar. Science of the Total Environment, 2021. 763: $\mathrm{p}$. 144204.

12. Zhou, Y., et al., Production and beneficial impact of biochar for environmental application: A comprehensive review. Bioresource Technology, 2021. 337: p. 125451.

13. Xie, Y., et al., A critical review on production, modification and utilization of biochar. Journal of Analytical and Applied Pyrolysis, 2022. 161: p. 105405.

14. Plácido, J., et al., Microalgae biochar-derived carbon dots and their application in heavy metal sensing in aqueous systems. Science of the Total Environment, 2019. 656: p. 531-539.

15. Barrientos, K., et al., Synthesis, Characterization and Ecotoxicity Evaluation of Biochar-Derived Carbon Dots from Spruce Tree, Purple Moor-Grass and African Oil Palm. Processes, 2021. 9(7): p. 1095.

16. Hidalgo, P., et al., Synthesis of carbon nanotubes using biochar as precursor material under microwave irradiation. Journal of environmental management, 2019. 244: p. 83-91.

17. Khalil, A., et al., Facile diazonium modification of pomegranate peel biochar: A stupendous derived relationship between thermal and Raman analyses. 2021.

18. Wang, J., et al., Efficient removal of fluoride using polypyrrole-modified biochar derived from slow pyrolysis of pomelo peel: sorption capacity and mechanism. Journal of Polymers and the Environment, 2018. 26(4): p. 1559-1572.

19. Endler, L.W., et al., Facile method to prepare biochar-NiO nanocomposites as a promisor material for electrochemical energy storage devices. Chemical Papers, 2020. 74(5): p. 1471-1476.

20. Qhubu, M.C., et al., Iron-Zinc Impregnated Biochar Composite as a Promising Adsorbent for Toxic Hexavalent Chromium Remediation: Kinetics, Isotherms and Thermodynamics. Chemistry Africa, 2021: p. 1-11.

21. Khalil, A.M., et al., Copper/nickel-decorated olive pit biochar: One pot solid state synthesis for environmental remediation. Applied Sciences, 2021. 11(18).

22. Huang, W.-H., D.-J. Lee, and C. Huang, Modification on biochars for applications: A research update. Bioresource Technology, 2021. 319: p. 124100.

23. Yang, Z., et al., Biochar nanocomposite derived from watermelon peels for electrocatalytic hydrogen production. ACS omega, 2021. 6(3): p. 2066-2073.

24. Jing, H., et al., Synthesis of $\mathrm{ZnO}$ nanoparticles loaded on biochar derived from spartina alterniflora with superior photocatalytic degradation performance. Nanomaterials, 2021. 11(10): p. 2479.

25. Yang, X., et al., Preparation and modification of biochar materials and their application in soil remediation. Applied Sciences, 2019. 9(7): p. 1365.

26. Cheng, B.-H., R.J. Zeng, and H. Jiang, Recent developments of post-modification of biochar for electrochemical energy storage. Bioresource technology, 2017. 246: p. 224-233.

27. Shang, L., et al., Adsorption of ammonium in aqueous solutions by the modified biochar and its application as an effective N-fertilizer. Water, air, \& soil pollution, 2018. 229(10): p. 1-15.

28. Li, F., et al., Use of polyacrylamide modified biochar coupled with organic and chemical fertilizers for reducing phosphorus loss under different cropping systems. Agriculture, Ecosystems \& Environment, 2021. 310: p. 107306.

29. Mirzaei, P., Préparation de matériaux d'électrode pour l'élimination et la valorisation de polluants azotés. 2018, Paris Est.

30. Liu, Y., et al., Metal-organic framework derived carbon-supported bimetallic copper-nickel alloy electrocatalysts for highly selective nitrate reduction to ammonia. Journal of Colloid and Interface Science, 2022.

31. Yao, D., et al., Graphene based copper-nickel bimetal nanocomposite: magnetically separable catalyst for reducing hexavalent chromium. ChemistrySelect, 2020. 5(11): p. 3243-3247.

32. Al-Enizi, A.M., et al., Copper nickel@ reduced graphene oxide nanocomposite as bifunctional electro-catalyst for excellent oxygen evolution and oxygen reduction reactions. Materials Letters, 2020. 260: p. 126969.

33. Ismail, M., et al., Green synthesis of plant supported CuAg and CuNi bimetallic nanoparticles in the reduction of nitrophenols and organic dyes for water treatment. Journal of Molecular Liquids, 2018. 260: p. 78-91.

34. Yuan, D., et al., Rational design of microporous biochar based on ion exchange using carboxyl as an anchor for high-efficiency capture of gaseous p-xylene. Separation and Purification Technology, 2021: p. 120402.

35. Sun, L., et al., Performance, kinetics, and equilibrium of methylene blue adsorption on biochar derived from eucalyptus saw dust modified with citric, tartaric, and acetic acids. Bioresource Technology, 2015. 198: p. 300-308. 
36. Shen, Y., N. Zhang, and S. Zhang, Catalytic pyrolysis of biomass with potassium compounds for Co-production of high-quality biofuels and porous carbons. Energy, 2020. 190: p. 116431.

37. Zabiszak, M., et al., Carboxyl groups of citric acid in the process of complex formation with bivalent and trivalent metal ions in biological systems. Journal of Inorganic Biochemistry, 2018. 182: p. 37-47.

38. Khemakhem, M. and M. Jaziri, Composites based on (ethylene-propylene) copolymer and olive solid waste: rheological, thermal, mechanical, and morphological behaviors. Polymer Engineering \& Science, 2016. 56(1): p. 27-35.

39. Puig-Gamero, M., et al., Obtaining activated biochar from olive stone using a bench scale high-pressure thermobalance. Journal of Environmental Chemical Engineering, 2021. 9(4): p. 105374.

40. Li, X., J.-i. Hayashi, and C.-Z. Li, Volatilisation and catalytic effects of alkali and alkaline earth metallic species during the pyrolysis and gasification of Victorian brown coal. Part VII. Raman spectroscopic study on the changes in char structure during the catalytic gasification in air. Fuel, 2006. 85(10-11): p. 1509-1517.

41. Pusceddu, E., et al., Comparison between ancient and fresh biochar samples, a study on the recalcitrance of carbonaceous structures during soil incubation. Int. J. New Technol. Res, 2017. 3: p. 39-46. 